\title{
Shock wave mixing in Einstein and dilaton gravity
}

\author{
Saurya Das ${ }^{a, 1}$, Parthasarathi Majumdar ${ }^{b, 2,3}$ \\ a The Instituic of Mathematical Sciences, CIT Campus. Madras - 600 113, India \\ b International Centre for Theoretical Physics, Trieste 1-34100, Italy \\ Received 22 November 1994 \\ Edilor: L. Alvarez-Gaumé
}

\begin{abstract}
We consider possible mixing of electromagnetic and gravitational shock waves, in the Planckian energy scattering of point particles in Minkowski space. By boosting a Reissner-Nordströı black hole solution to the velocity of light, it is shown that no mixing of shock waves takes place for arbitrary finite charge carried by the black hole. However, a similar boosting procedure for a charged black hole solution in dilaton gravity yields some mixing the wave function of even a neutral test particle, acquires a small additional phase factor depending on the dilatonic black hole charge. Possible implications for poles in the amplitudes for the dilaton gravity case are discussed.
\end{abstract}

\section{Introduction}

The predominance of shock waves as instantaneous mediators of gauge and gravitational interactions in two particle scattering at Planckian centre of mass energies and fixed low momentum transfers has been the subject of some interest recently [1-5]. All purely local gauge and/or gravitational field degrees of freedom decouple in this kinematical regime. The residual degrees of freedom are basically gauge or coordinate transformation parameters evaluated on the boundary of the null plane, describing a simpler field theory whose classical solutions represent appropriate shock waves. Two particle $S$-matrices are exactly calculable from these reduced theories and reproduce, wherever possible, the corresponding eikonal amplitudes

\footnotetext{
1 E-Mail: saurya@imsc.ernet.in.

${ }^{2}$ E-Mail: partha@ictp.trieste.it; partha@imsc.ernet in.

${ }^{3}$ Permanent address: The Institute of Mathematical Sciences, Madras 600113 ; India.
}

$[3,10]$. There are of course situations when this decoupling of local degrees of freedom is not easy to establish within a field theory, as e.g., when magnetic monopoles are present. However, this does not deter description of the ensuing interactions in terms of shock waves, nor does it in any way affect the computation of two particle amplitudes. The interplay between electromagnetic anil gravitational interactions is particularly significant in this case since the magnetic monopole sector is basically strongly coupled, akin to gravity at Planckian energies [5].

The gravitational shock wave relevant to two particle scattering in Minkowski space has been obtained [6] in one of two ways: either by demanding that the Minkowskian geometry with a lightlike particle present is the same as an empty Minkowski space with coordinates shifted along the geodesic of the particle, or by a process of 'boosting' the metric of a massive particle to luminal velocities when its mass exponentially decays to zero. Since there are well known (electrically and magnetically) charged black hole so- 
lutions of the Einstein squation, boosting such solutions to the velocity of light would of course produce both gravitational and electromagnetic shock waves: It is then imporiant to determine whether these two species of shock waves actually mix. The problem may be stated succinctly as follows: the calculation of the amplitude in the shock wave picture entails computing the phase frctor that the shock wave induces on the wave function of the target particle. When both particles carry charge, phase factors are induced by electromagnetism and gravity independently of the other. It has been assumed in the literature that, with both shock waves present, the net phase factor is simply the sum of the individual phase factors $[1,3,5]$. In other words, the gravitational and electromagnetic shock waves are assumed to travel collinearly without interaction, even though they are extremely localized singulai field configurations. In our opinion, this assumption warrants justification. This is what is attempted in the sequel.

The study of mixing of the two varieties of shock waves proceeds by considering black hole solutions that also carry electric/magnetic charge, and as such, produce both kinds of shock waves upon boosting a la Dray and 't Hooft [6]. In Section II we show that a boosting of the standard Reissner-Nordstrom black hole metric leads to a decoupling of electromagnetic and gravitational effects in a somewhat subtle manner: the part of the boosted metric that depends explicitly on the charge, san be removed by a diffeomorphism, leaving behind a piece which cannot, being non-differentiable at $x^{-}=0$. This discontinuity' in the boosted metric, which constitutes the gravitational shock wave, is identical to the discontinuity in the boosted Schwarschild metric, independent of the charge of its parent black hole solution. In the next section, we attempt an application of a similar boosting procedure to charged black hole solutions of four dimensional dilaton gravity. For generic values of the charge, this endeavor results in a boosted metric whose discontinuities do explicitly depend on the charge. More importantly, in this case both null coordinates appear to warrant a discontinuous transformatien. We discuss the implication of this in terms of singularities of the metric, and show that the gravitational phase shift of the wave function of a test particle depinds on the black hole charge even when the particle isself is neutral. We end in Secion it with a few concluding remarks.

\section{Decoupling in the Reissner-Nordström case}

The gravitational field due to a stationary point particle of mass $M$ and electric charge $Q$ is given by the standard Reissner-Nordström metric

$$
\begin{aligned}
d s^{2} & =\left(1-\frac{2 G M}{r}+\frac{G Q^{2}}{r^{2}}\right) d t^{2} \\
& \left(1-\frac{2 G M}{r}+\frac{G Q^{2}}{r^{2}}\right)^{-1} d r^{2}-r^{2} d \Omega^{2}
\end{aligned}
$$

where $G$ is Newton's constant. The question we address here is: if the particle is Lorentz-boosted to a velocity $\beta \sim 1$, what will be the nature of the gravitational field as observed in the 'stationary' frame? Let us assume, for simplicity and without loss of generality, that the particle is boosted in the $+z$ direction, so that $z, t$ are related to the tranformed coordinates $Z, T$ according to

$$
\begin{aligned}
& T=t \cosh \rho+z \sinh \rho \\
& Z=t \sinh \rho+z \cosh \rho .
\end{aligned}
$$

The parameter $\rho$ is called rapidity: $\beta=\tanh \rho$. The null coordinates are defined as usual as $x^{ \pm}=t \pm z$. The boosting involves parametrizing the mass of the black hole as $M=2 p e^{-\rho}$ where $p$ is the momentum of the boosted particle, lying almost entirely in the longitudinal direction. $p$ is usually kept fixed at a large value in the boosting process, and the limit of the boosted metric is evaluated as $\rho \rightarrow \infty$. In this limit, the Reissner-Nordström metric assumes the form

$$
\begin{aligned}
& \left.d s^{2} \rightarrow d x\left\{d x^{+}-d x-\frac{2 G p}{|x-|}-\frac{G Q^{2}}{\left(x^{-}\right)^{2}}\right]\right\} \\
& -d x_{\perp}^{2} .
\end{aligned}
$$

The boosted metric does indeed seem to depend explicitly on the charge $Q$. But, notice that this depenJence is confined to a part of the metric that can be remove, by a diffenorphism, albeit one that is singular at the origin. However, the part that goes as $1 /\left|x^{-}\right|$ cannot be removed by any diffeomorphism; this latter, of course, is precisely the part that is associated with the gravitational shock wave [1]. Further not orly is its coefficient independent of $Q$, it is identical to the the coefficient of the $1 /\left|x^{-}\right|$term in the boosted Schwarschild metric $[1,6]$. All memory of the charge of the parent black hole solution is obliterated upon 
boosting, insofar as the gravitational shock wave is concerned. Consequently, the mutual transparency of the two shock waves follows immediately: ${ }^{4}$ Hence the net phase shift of the wave function of a test particle moving in the two shock waves is simply the sum of the phase factors induced individually by each shock wave. This, in the case of scattering of two electric charges, simply amounts to the replacement $G s \rightarrow G s+e e^{\prime}$ as mentioned in Refs [1,3]. A similar decoupling of electromagnetic and gravitational shock waves can be seen by boosting a magnetically charged Reissner-Nordström solution, which justifies once again the determination of the net phase factor in the test charge wave function as the sum of the individual phase factors in Planckian charge-monopole scattering [5].

The foregoing analysis is completely general; and requires no assumption on the strength of the charge $Q$, except perhaps that it be finite. However, an analysis of the singularities of the the metric in (1) indicates that, if one is to abide by the dictates of Cosmic Censorship, the charge $Q$ must obey $Q \leq M$. In the extremal limit, the boosting procedure adopted above forces $Q$ to decay exponentially to zero as the rapidity runs to infinity. The electromagnetic shock wave, by withering away in this limit, then trivially decouples from the gravitational one. This was first pointed out in Ref. [7].

\section{Non-decoupling in dilaton gravity}

The charged black hole solution of four dimensional dilaton gravity, obtained as a part of an effective low energy theory from the heterotic string compactified on some compact six-fold, is given, following $[8,9]$. as 5

$$
\begin{aligned}
& d s^{2}=\left(1-\frac{\alpha}{M r}\right)^{-1}\left[\left(1-\frac{2 G M}{r}\right) d t^{2}\right. \\
& \left.-\left(1-\frac{2 G M}{r}\right)^{-1} d r^{2}-\left(1-\frac{\alpha}{M r}\right) r^{2} d \Omega^{2}\right]
\end{aligned}
$$

\footnotetext{
${ }^{4}$ We note here that our approach and results for the ReissnerNordström case differ somewhat from those of Ref. [7] where, in fact, the limiting procedure employed appears to yield vanishing electromagnetic shock waves in the luminal limit.

5. This metric is the so-called string metric $[9]$. What follows is equally valid for the Einstein metric
}

where, $\alpha \equiv Q^{2} e^{-2 \phi_{0}}$, with $\phi_{0}$ being the asymptotic value of the dilaton ield $\phi$. The metric reduces to the Schwarzschild metric when $\alpha=0$, and, not surprisingly, shares the coordinate singularity at $r=2 G M$ which becomes the event horizon for the curvature singularity at $r=0$. In addition, there is the 'singularity' at $r=\alpha / M$ which is not necessarily a coordinate singularity. We shall return to this point later.

We now apply the boosting procedure elaborated in the last section to this metric. The mass of the black hoie is parametrized as $M=2 p e^{-\rho}$ and the Lorentztransformed metric is evaluated in the limit as the rapidity $\rho \rightarrow \infty$ for fixed large $p$. The result can be expressed as the Minkowski metric in terms of shifted coordinate differentials,

$$
d s^{2} \rightarrow d \tilde{x}^{\prime} d \tilde{x}^{-}-\left(d \tilde{x}_{\perp}\right)^{2}
$$

where,

$$
\begin{aligned}
& d \tilde{x}^{+}=d x^{+}-\left(\frac{\frac{4 G p}{\mid x-1}}{1-\frac{\alpha}{p \mid x^{-}}}\right) d x^{-} \\
& d \tilde{x}^{-}=d x^{-}\left(\frac{1-\frac{\alpha}{2 p \mid x-1}}{1-\frac{\alpha}{p \mid x-1}}\right) \\
& d \tilde{\boldsymbol{x}}_{\perp}=d \boldsymbol{x}_{\perp} .
\end{aligned}
$$

Several features emerge immediately from these equations; of these, the most striking is the explicit dependence on the charge $\alpha$ of terms that will surely contribute to the gravitational shock wave because of their non-differentiable functional form. No less important is the fact that, in this case, the coordinatc $x^{-}$which, in the Schwarschild (and Reissner-Nordström) case(s), defined the null surface $\left(x^{-}=0\right)$ along which the two Minkowski spaces were to be glued, is now itself subject to transformation by such a discontinuous function, again explicitly depending on $\alpha$. Before examining these aspects in detail, we note in passing that the results reduce to those in the Schwarschild case in the limit $\alpha=0$, as indeed is expected.

First of all, the charge $\alpha$ may be chosen to be sinall by taking a large value of $\phi_{0}$, so that, with a large value of $p$, one can binomially expand the denominators in the rhs of the first two equations in (5); this yields, for points away from $x^{-}=0$, 


$$
\begin{aligned}
& d \tilde{x}^{+}=d x^{+}-\left[\frac{4 G p}{|x-|}+\frac{4 \alpha}{\left(x^{-}\right)^{2}}\right] d x^{-}+\mathcal{O}\left(\alpha^{2} / p\right) \\
& d^{+}=d x+\frac{\alpha}{2 p|x|} d x+\mathcal{O}\left(\alpha^{2} / p^{2}\right)
\end{aligned}
$$

We now observe that, as far as the shift in $d x^{+}$is concerned, the part that will contribute to the gravitational shock wave is in fact, independent of $c$, and, furthermore, is identical to the result in the Schwarschild and hence the Reissner-Nordström case. As for the latter solution, the $\alpha$-dependent part may be rendered innocuous by a smooth diffeomorphism.

This is however not the case for the shift in $d x^{-}$, which is explicitly $\alpha$-dependent. Clearly, the gravitational shock wave now possesses a more complicated geometrical structure than in the earlier examples. The geometry can no longer be expressed as two Minkowski spaces glued after a shift along the null surface $x^{-}=0$, for now there is a discontinuity in the $x^{-}$coordinate at that very point, in contrast to the previous cases where it was continuous. This discontinuity has a rather serious implication: unlike in the earlier situation wherein the coordinate $x^{-}$could well serve as the affine parameter characterising the nuil geodesic of a test particle crossing the gravitational shock wave (cf. [6]), a null geodesic is actually incomplete in this situation. To see this in more detail, consider the geodesic equations of a very light particle moving in the Lorentz-boosted meiric (4),

$$
\begin{aligned}
& T=\left(\frac{1-\frac{\alpha}{M r}}{1-\frac{2 G M}{r}}\right) E \\
& r^{2} \dot{\phi}=\left(1-\frac{2 G M}{r}\right) L \\
& \dot{r}^{2}=\left(1-\frac{\alpha}{M r}\right)^{2}\left[E^{2}-\frac{L^{2}}{r^{2}}\left(\frac{1-\frac{2 G M}{r}}{1-\frac{\alpha}{M r}}\right)\right] .
\end{aligned}
$$

Unlike the geodesic equations for a boosted Schwarzschild metric, which can be solved perturbatively in a power series in the mass $M$ (or alternatively in the parameter $e^{-p}$ (where $\rho$ is the rapidity), [6] these equations do not admit any perturbative solution hecause of the singularity at $r=\alpha / M$. Taking recourse to singular perturbation theory does not evade the problem; the definition of a continuous affine paramcer is not possible in this case. It follows that the singularity in question must be a curvature singularity. Although for the Reissner-Nordström case also, for generic value of the charge, the singularity at $r=0$ is no longer hidden by the event horizon, there are no other singularities away from this point. In the present instance, the singularity at $r=0$ is actually protected by the Schwarzschild horizon. One might consider imposing an extremal condition on the charge $\alpha$ (vid. [9] ): $\alpha=2 M^{2}$ to mitigate the circumstances. However, this limit is not interesting for our purpose, for the same reason that the extremal Reissner-Nordström is not - the charge decays exponentially to zero with the rapidity going off to infinity.

The non-decoupling of gravitational and electromagnetic effects that we see here can be made more articulate if one prweeds to actually calculate the phase shift of the wave function of a test particle encountering the gravitational shock wave, notwithstanding the pathologies delineated above. The Eqs. (5) above for the differentials are consistent with the following finite shifts, obtained by generaliaing results of [6],

$$
\begin{aligned}
& x_{>}^{+}=x_{<}^{+}+2 G p \ln \mu^{2} r_{\perp}^{2} \\
& x_{>}^{-}=x_{<}^{-}+\frac{\alpha}{2 p} \ln \mu^{2} r_{\perp}^{2} \\
& x_{>}=x_{<}
\end{aligned}
$$

With these, following [1] we can easily calculate the nct phase shift of the wave function of a test particle due purely to gravitational effects:

$\Phi_{\text {total }}=\left(G s+\alpha \frac{k_{\perp}^{2}}{2 s}\right) \ln \mu^{2} r_{\rfloor}^{2}$

Here, $k_{\perp}$ is the transverse momentum of the test particle. Thus, even if the test particle is electromagnetically neutral, its wave function undergoes a phase shift that depends un the charge of the black hole boosted to produce the gravitational shock wave. This is a novel phenomenon, in our opinion, although, strictly speaking, in the kinematical regime under consideration, the magnitude of the effect is small. Nevertheless, the mixing of the electromagnetic and gravitational shock waves, in this case is quite obvious.

The scattering amplitude for a test particle encountering such a gravitational shock wave can be calculated following Ref. [1]. Modulo standard kinematical factors and irrelevant constants, the answer is 
$f(s, t) \sim \frac{1}{t} \frac{\Gamma\left(1-i\left(G s+\alpha \frac{k_{\perp}^{2}}{2 s}\right)\right)}{\Gamma\left(i\left(G s+\alpha \frac{k_{\perp}^{2}}{2 s}\right)\right)}$

Since the calculation is performed in a coordinate frame in which the test particle is assumed to be moving slowly, the amplitude does not appear manifestly Lorentz-invariant, although there is nowhere any violation of Lorentz symmetry. A more refined calculation where I-orentz invariance is explicitly maintained can indeed be done following Verlinde and Verlinde [2], but will not be reported here. The only likely outcome of such a calculation will be the replacement of the quantity $k_{\perp}^{2}$ by the squared momentum transfer $t$ upto somc numerical coefficient of $\mathcal{O}(1)$. As a consequence, the poles in (11) would undergo a shift of $\mathcal{O}\left(i \alpha G t / N^{2}\right)$ from their integer-valued (given by $N$ ) positions on the imaginary axis found in the Schwarzschild case [1]. This shift is quite different from similar shifts when clectromagnetic effects are included based upon a decoupling assumption $[1,3,5]$. The non-decoupling is manifest from the coefficient $\alpha G$ in this case. Also, the electromagnetic shifts are always constant independent of $t$, in contrast to what we find here.

\section{Conclusions}

The decoupling of electromagnetic and gravitational shock waves have now been established for the case of general relativity, justifying thereby earlier results incorporating both fields for electrically and magnetically charged particles scattering at Planckian centreof-mass energies. The shifts in the poles due to electromagnetic effects stand vindicated. Admittedly, it is true that in the Einstein gravity case the poles appear to be artifacts of the large impact parameter approximation [2]. But, the nature of the shift due to the dilaton coupling tends to reinforce the speculation that string theory may actually provide a way to compute corrections to this approximation as a power series in $t$. It would be interesting if this behaviour could be retrieved from the high energy string amplitudes calculated in earlier work [10] in some suitable local field theory limit.

The results may also have implications for blark holes. The effect of infalling particles collapsing grav- itationally onto a black hole has been analyzed $[6]$ to produce a shift of the classical event horizon. If we also subscribe to the view [11] that this shift essentially involves generalizing the flat space gravitational shock wave to a curved background, then a particle whose fields are obtained by boosting fields of a dilaton black hole would cause extra shifts of the horizon of a Schwarzschild black hole. In addition, with electric and magnetic charges present, novel contributions are to be expected for any $S$-matrix (a la 't Hcoft [11]) one may attempt to propose for dilatonic black holes.

\section{Acknowledgements}

We thank T. Jayaraman, R. Shankar and K. Subramaniam for useful discussions, and $R$. Basu for a careful reading of the manuscript. One of us (P.M.) also thanks F. Hussain, S. Mukherjee, M. A. Namazie, K. Narain, S. Randjbar-Daemi and G. Thompson for very fruitful discussions, and the International Centre for Theoretical Physics, Trieste, Italy for excellent hospitality during an Associateship visit uring which this work was completed.

\section{Note added}

As already noted, the authors in Ref. [7] have also found the shock wave geometry due to an ultrarelativistic charged particle. However, for this purpose, in addition to the the mass; they have also parametrized its charge $Q$ in terms of the rapidity $\rho$ such that as $\rho \rightarrow \infty, Q \rightarrow 0$, but the electromagnetic energy moment tensor has non-vanishing components. Consequently, for thein, the electromagnetic and gravitational shock waves do not decouple in the ReissnerNordstrom case. We prefer instead to study the question of decoupling of the two species of shock waves for arbitrary fixed electric charge $Q$, following a naive extension of the direct boosting procedure adopted in Ref. [6]: This is done both for the Reissner-Nordstrom and the dilaton gravity cases. It does not seem imperative to use a parametrization for the charge similar to the mass in this procedure, except in the extremal case where the charge is restricted by the mass. If we were to use such a charge rescaling for the case of the 
dilaton gravity, the results would indeed change quantatively, ulthough the main qualitative outcome, viz., a neutral test particle being able to 'sense' the charge of the luminally boosted black hole, stands. We hasten to add, however, the above conclusions are only to be taken seriously within the very restricted kinematical domain of large $s$ and vanishingly small fixed t. Further, the heuristic analysis presented here must eventually be supplanted by more rigorous ones. An attempt in this direction is in progress [12].

After completion of this paper, we became aware of the work of K. Sfetsos [13] wherein the gravitational fields due to a boosted neutral particle in ReissnerNordstrom and dilatonic black hole backgrounds have been obtained directly from the relevant field equations. However, the issue that we address in this letter, namely the decoupling of shock waves, has not been considered there explicitly.

\section{References}

[i] G. 't Hooft. Phys, Leit. B 198 (1987) 61; Nucl. Phys. B 304 (1988) 867

[2] H. Verlinde and E. Verlinde, Nucl, Phys. B 37 ( 1992) 246.

[3] R. Jackiw, D. Kabat and M. Ortiz, Phys Lett B 277 ( 1992) 148.

14] V. Verlinde and E. Verlinde. Princeton University preprint PJPT-1319 (1993)

[5! S. Das and P. Majumdar, Phys. Rev. Lett. 72 (1994) 2524; ICTP-IMSc preprint IC/94/346, IMSc-94/44, hep-th 9411061.

[6] T. Dray and G. 't Hooft, Nucl. Phys. B 253 (1985) 173.

[7] C. Lousto and N Sánchez, Int. J. Mod. Phys. A 5 (1990) 915.

[8] G. Gibbons and K. Maeda, Nucl. Phys. B 298 (1988) 74l.

[9] H. Garfinkle, G. Horowitz and A. Strominger, Phys. Rev. D 43 (1991) 3140; D 45 (1992) 3888(E).

[10] D. Amati, SISSA preprint SISSA-22-93-EP, and references therein.

[11] G. 't Hooft, Nucl. Phys. B 335 (1990) 138.

[12] S Das, to appear

[13] K. Sfetsos, On Gravitational Shock. Waves in Curved Spacetimes, Utrecht preprint THU-94/13 (August 1994), hep-th/9408169. 9408129. 